\title{
Nonexistence of proper holomorphic maps between certain classical bounded symmetric domains
}

\author{
Ngaiming Mok*
}

\begin{abstract}
The author, motivated by his results on Hermitian metric rigidity, conjectured in Mok [Mk2, 1989] that a proper holomorphic mapping $f: \Omega \rightarrow \Omega^{\prime}$ from an irreducible bounded symmetric domain $\Omega$ of rank $\geq 2$ into a bounded symmetric domain $\Omega^{\prime}$ is necessarily totally geodesic provided that $r^{\prime}:=\operatorname{rank}\left(\Omega^{\prime}\right) \leq \operatorname{rank}(\Omega):=r$. The Conjecture was resolved in the affirmative by Tsai [Ts, 1993]. When the hypothesis $r^{\prime} \leq r$ is removed, the structure of proper holomorphic maps $f: \Omega \rightarrow \Omega^{\prime}$ is far from being understood, and the complexity in studying such maps depends very much on the difference $r^{\prime}-r$, which we call the rank defect. The only known nontrivial non-equidimentional structure theorems on proper holomorphic maps are due to $\mathrm{Tu}$ [Tu2, 2002], in which a rigidity theorem was proven for certain pairs of classical domains of type I, which implies nonexistence theorems for other pairs of such domains. For both results the rank defect is equal to 1, and a generalization of the rigidity result to cases of higher rank defects along the line of arguments of [Tu2] has so far been inaccessible. In this article we produce nonexistence results for infinite series of pairs of $\left(\Omega, \Omega^{\prime}\right)$ of irreducible bounded symmetric domains of type I in which the rank defect is an arbitrarily prescribed positive integer. Such nonexistence results are obtained by exploiting the geometry of characteristic symmetric subspaces as introduced by Mok-Tsai [MT, 1992] and more generally invariantly geodesic subspaces as formalized in Tsai [Ts]. Our nonexistence results motivate the formulation of questions on proper holomorphic maps in the non-equirank case.
\end{abstract}

Keywords: Proper holomorphic maps, bounded symmetric domains, characteristic symmetric subspaces, invariantly geodesic subspaces, rank defects

2000 MR Subject Classification: 32M15, 32H35

E. Cartan introduced series of irreducible domains which are now known as classical domains. They break down into four series, the first three of which being defined in terms of matrices. On top of these series it was later found that there are two additional irreducible bounded symmetric domains pertaining to exceptional Lie groups. Any bounded symmetric domain admits a canonical bounded realization given by the HarishChandra embedding, and in the case of the classical domains the canonical bounded realizations agree with those given by E. Cartan.

Bounded symmetric domains and their compact quotients and more generally quotients of finite volume with respect to canonical metrics are objects of study with a vast literature. From the geometric perspective one central theme is the notion of rigidity. In this direction Siu [Si, 1978] discovered the $\partial \bar{\partial}$-Bochner-Kodaira formula and applied it to study the strong rigidity of compact quotients of irreducible bounded symmetric domains. For holomorphic mappings by the work of Mok [Mk1, 1987] on Hermitian metric rigidity it follows that a nonconstant holomorphic mapping $f: X \rightarrow X^{\prime}$ between quotients of irreducible bounded symmetric domains must be totally geodesic, provided that the domain manifold $X$ is of rank $\geq 2$ and of finite volume. If the domain manifold

* Research partially supported by a CERG of the Research Grants Council of Hong Kong, China 
$X$ is compact and the homomorphism $f_{*}: \pi_{1}(X) \rightarrow \pi_{1}\left(X^{\prime}\right)$ is injective, then the lifted holomorphic map $F: \Omega \rightarrow \Omega^{\prime}$ on uniformizing bounded symmetric domains is a proper holomorphic map. Motivated in part by Hermitian metric rigidity, the author formulated a conjecture in Mok [Mk2, 1989; Chapter 6, (5.3)] according to which any proper holomorphic mapping $f: \Omega \rightarrow \Omega^{\prime}$ between bounded symmetric domains is necessarily totally geodesic provided that $\Omega$ is irreducible and of $\operatorname{rank} \geq 2$, and $\operatorname{rank}\left(\Omega^{\prime}\right) \leq \operatorname{rank}(\Omega)$. The Conjecture was resolved in the affirmative by Tsai [Ts, 1993].

In what follows we restrict our attention to the case of proper holomorphic maps defined on irreducible bounded symmetric domains $\Omega$ of rank $\geq 2$. After the work of Tsai $[\mathrm{Ts}]$ it remains to understand proper holomorphic maps $f: \Omega \rightarrow \Omega^{\prime}$ where $r:=\operatorname{rank}(\Omega)<\operatorname{rank}\left(\Omega^{\prime}\right):=r^{\prime}$. We will call this the non-equirank case, and the difference $r^{\prime}-r$ will be called the rank defect. In the non-equirank case the only known rigidity result so far is that of $\mathrm{Tu}$ [Tu2, 2002], where he found examples of Cartan domains $\Omega, \Omega^{\prime}$ of type I where any proper holomorphic map $f: \Omega \rightarrow \Omega^{\prime}$ is necessarily totally geodesic. In this set of examples the rank defect is always equal to 1 . From such a rigidity result Tu deduced also some nonexistence results for proper holomorphic maps $f: \Omega \rightarrow \Omega^{\prime}$ where $\Omega$ and $\Omega^{\prime}$ are again Cartan domains of type $\mathrm{I}$, and where the rank defect is also 1 .

In this article we examine further nonexistence results for proper holomorphic maps $f: \Omega \rightarrow \Omega^{\prime}$, where to focus on the discussion we will restrict ourselves to Cartan domains of type I. We will show in this context that there are nonexistence results generalizing those of $\mathrm{Tu}[\mathrm{Tu} 2]$ in which the rank defect is arbitrarily large. More precisely, given any positive integer $\ell$, we prove that there exist infinite series of pairs of Cartan domains $\left(\Omega, \Omega^{\prime}\right)$ of type I such that $2 \leq \operatorname{rank}(\Omega)<\operatorname{rank}\left(\Omega^{\prime}\right)$, and such that the rank defect is equal to $\ell$, for which there exist no proper holomorphic mappings $f: \Omega \rightarrow \Omega^{\prime}$. The main tool of our argument goes back to the use of characteristic symmetric subspaces of Mok-Tsai [MT, 1992] and invariantly geodesic subspaces as formulated in Tsai [Ts], and a study of meromorphic maps on moduli spaces of such subspaces induced from $f: \Omega \rightarrow \Omega^{\prime}$. The complexity in the study of proper holomorphic maps in the nonequirank cases increases with the rank defect, and our nonexistence result also serves to motivate a search for rigidity results with arbitrarily large rank defects.

\section{$\S 1$ Background notions and results}

(1.1) Let $\left(X_{0}, g_{0}\right)$ be an irreducible Hermitian symmetric space of the noncompact type and $\left(X_{c}, g_{c}\right)$ be its dual Hermitian symmetric space of the compact type. Write $X_{0}=$ $G_{0} / K, X_{c}=G_{c} / K$; where $G_{0}$ resp. $G_{c}$ is the identity component of the isometry group of $\left(X_{0}, g_{0}\right)$ resp. $\left(X_{c}, g_{c}\right)$, and $K \subset G_{0}$ resp. $K \subset G_{c}$ is an isotropy subgroup at any reference point $0 \in X_{0}$ resp. $0 \in X_{c}$. By a characteristic vector we mean a nonzero highest weight vector of the isotropy representation of $K$ on $T_{0}\left(X_{0}\right)$ resp. $T_{0}\left(X_{c}\right)$. In Mok-Tsai $[\mathrm{MT}, 1992]$ we introduced the notion of (Hermitian) characteristic symmetric subspaces of $\left(X_{0}, g_{0}\right)$ resp. $\left(X_{c}, g_{c}\right)$. The definition given in [MT, (1.4)] was formulated in Lie-theoretic terms, in terms of subsets of a maximal strongly orthogonal set of noncompact positive roots. We give here a more geometric equivalent definition, 
as follows. Here and in what follows a (sub)manifold is understood to be connected.

Let $P \subset X_{0}$ be a maximal polydisk. In other words, writing $r$ for the rank of $\left(X_{0}, g_{0}\right), P$ is biholomorphic to the $r$-dimensional polydisk $\Delta^{r}$, and $\left(P,\left.g_{0}\right|_{P}\right) \hookrightarrow\left(X_{0}, g_{0}\right)$ is a totally geodesic complex submanifold. Here and henceforth the term 'polydisk' includes the case of the disk. When $r \geq 2$ let $k$ be an integer such that $1 \leq k \leq r-1$, and write $P=\Pi^{\prime} \times \Pi^{\prime \prime}$ for any decomposition of $P$ into a Cartesian product of polydisks $\Pi^{\prime}$ and $\Pi^{\prime \prime}$ such that $\operatorname{dim}\left(\Pi^{\prime}\right)=k, \operatorname{dim}\left(\Pi^{\prime \prime}\right)=r-k$. We have $\left(P,\left.g_{0}\right|_{P}\right) \cong\left(\Pi^{\prime}, g^{\prime}\right) \times\left(\Pi^{\prime \prime}, g^{\prime \prime}\right)$ as Kähler manifolds, where $\left(\Pi^{\prime}, g^{\prime}\right)$ is the $k$-fold product of a Poincaré disk $\left(\Delta, d s_{\Delta}^{2}\right)$ and $\left(\Pi^{\prime \prime}, g^{\prime \prime}\right)$ is the $(r-k)$-fold product of $\left(\Delta, d s_{\Delta}^{2}\right)$. For $x \in P$ let $\mathcal{N}_{x} \subset T_{x}\left(X_{0}\right)$ denote the complex vector subspace defined by $\mathcal{N}_{x}:=\left\{\zeta \in T_{x}\left(X_{0}\right): R_{\eta \bar{\eta} \zeta \bar{\zeta}}=0\right.$ for every $\eta \in$ $\left.T_{x}\left(\Pi^{\prime}\right)\right\}$. Then, by means of the Jacobi identity and Lie triple systems it follows readily that $\mathcal{N}_{x}$ is tangent to a totally geodesic complex submanifold $S_{x}$ of $X_{0}$. All the $S_{x}$ are biholomorphic to one another, and we have a totally geodesic holomorphic isometric embedding $\tau:\left(\Pi^{\prime}, g^{\prime}\right) \times(S, h) \rightarrow\left(X_{0}, g_{0}\right)$ for some Hermitian symmetric space $(S, h)$ such that for $x \in P$ we have $S_{x}=\tau\left(\left\{y^{\prime}\right\} \times S\right)$ for some $y^{\prime} \in \Pi^{\prime}$. This gives the notion of characteristic symmetric subspaces of $\left(X_{0}, g_{0}\right)$, as follows.

Definition 1. Let $\left(X_{0}, g_{0}\right)$ be an irreducible Hermitian symmetric space of the noncompact type and of rank $r \geq 2$. Let $P \subset X_{0}, P \cong \Delta^{r}$, be an arbitrary maximal polydisk. Let $k$ be an integer such that $1 \leq k \leq r-1$, and $P=\Pi^{\prime} \times \Pi^{\prime \prime}$ be a decomposition of $P$ into a Cartesian product of polydisks $\Pi^{\prime}$ and $\Pi^{\prime \prime}$, where $\operatorname{dim}\left(\Pi^{\prime}\right)=k, \operatorname{dim}\left(\Pi^{\prime \prime}\right)=r-k$. By characteristic symmetric subspaces we mean the totally geodesic complex submanifolds $S_{x} \subset X_{0}$ constructed as described above for any choice of $P \subset X_{0}$, any choice of decomposition $P=\Pi^{\prime} \times \Pi^{\prime \prime}$ and any choice of $x \in P$.

The definition of characteristic symmetric subspaces of an irreducible Hermitian symmetric space $\left(X_{c}, g_{c}\right)$ is formally identical to the definition in the case of the noncompact type provided that we replace maximal polydisks by maximal polyspheres. It turns out that every characteristic symmetric subspace (in both the cases of the noncompact type and the compact type) is irreducible, and all characteristic symmetric subspaces of the same rank are equivalent to each other under automorphisms of $\left(X_{0}, g_{0}\right)$ resp. $\left(X_{c}, g_{c}\right)$.

For the case of the noncompact type $\left(X_{0}, g_{0}\right)$, realized as a bounded domain $\Omega \Subset \mathbb{C}^{N}$ by means of the Harish-Chandra embedding, there is in addition the notion of boundary components (cf. Wolf [Wo]). In the case of the noncompact type a characteristic symmetric subspace $S \subset X_{0}$ is equivalently a totally geodesic complex submanifold biholomorphic to some positive-dimensional boundary component $\Phi \subset \partial \Omega$ in such a way that characteristic vectors of $S$ (which is known to be irreducible) as an abstract irreducible Hermitian symmetric space of the noncompact type are characteristic vectors on $X_{0}$.

In the case of the compact type characteristic symmetric subspaces are examples of invariantly geodesic subspaces, a notion implicit in Mok-Tsai [MT] and formally defined in Tsai $[\mathrm{Ts}]$. (Here to conform with proceeding terminology we use the term 'subspace' in place of 'submanifold'.) We recall its definition, as follows. 
Definition 2 (Tsai [Ts, $\S$ 4, Definition 4.1]). Let $\left(X_{c}, g_{c}\right)$ be a Hermitian symmetric space of the compact type. A complex submanifold $M \subset X_{c}$ is said to be an invariantly geodesic subspace if and only if $M$ is totally geodesic in $\left(X_{c}, \varphi^{*} g_{c}\right)$ for any biholomorphic automorphism $\varphi \in \operatorname{Aut}\left(X_{c}\right)$.

For the case of a Hermitian symmetric space $\left(X_{0}, g_{0}\right)$ of the noncompact type, the automorphism group agrees with the group of holomorphic isometries with respect to the Bergman metric, and the analogue of Definition 2 does not apply. In this article we extend the terminology to apply to the case of the noncompact type by making use of the Borel embedding $X_{0} \subset X_{c}$ for a dual pair $\left(X_{0}, X_{c}\right)$ of Hermitian symmetric spaces of the compact and the noncompact types, as follows.

Definition 3. Let $\left(X_{0}, g_{0}\right)$ be a Hermitian symmetric space of the noncompact type, $\left(X_{c}, g_{c}\right)$ be its dual Hermitian symmetric space of the compact type, and $X_{0} \subset X_{c}$ be the Borel embedding. A complex submanifold $S \subset X_{0}$ is said to be an invariantly geodesic subspace if and only if there is an invariantly geodesic subspace $M \subset X_{c}$ such that $M$ contains $S$ as an open subset.

We observe that $\left(S,\left.g_{0}\right|_{S}\right)$ is totally geodesic in $\left(X_{0}, g_{0}\right)$. To see this consider the dual pair $\left(X_{0}, g_{0}\right)$ and $\left(X_{c}, g_{c}\right)$ of Hermitian symmetric spaces of the noncompact resp. compact type. Let $X_{0} \cong \Omega \Subset \mathbb{C}^{N} \subset X_{c}$ be the Harish-Chandra and Borel embeddings of $X_{0}$, where $X_{0}$ is identified with the bounded symmetric domain $\Omega$. For any $s \in S$, there exists $\varphi \in \operatorname{Aut}\left(X_{0}\right) \subset \operatorname{Aut}\left(X_{c}\right)$ such that $\varphi(s)=0$. Since by the hypothesis $M \subset X_{c}$ is an invariantly geodesic subspace, replacing $M$ by $\varphi(M)$ without loss of generality we may assume that $0 \in S \subset M$ and that the arbitrary base point is the origin $0 . M$ is in particular totally geodesic with respect to $g_{c}$. At $0 \in \mathbb{C}^{N}$ the RiemannChristoffel symbols of both $\left(X_{c}, g_{c}\right)$ and $\left(X_{0}, g_{0}\right)$ vanish due to Riemannian symmetry, and the total geodesy of $\left(M,\left.g_{c}\right|_{M}\right)$ in $\left(X_{c}, g_{c}\right)$ implies that the second fundamental form of $\left(S,\left.g_{0}\right|_{S}\right)$ in $\left(X_{0}, g_{0}\right)$ vanishes at $0 \in S$. Since any point on $S$ can be taken to be the origin 0 it follows that $\left(S,\left.g_{0}\right|_{S}\right)$ is totally geodesic in $\left(X_{0}, g_{0}\right)$, as observed. Beyond this observation the terminology in Definition 3 may appear ad hoc as the notion of invariantly geodesic subspaces for $\left(X_{c}, g_{c}\right)$ refers to canonical Kähler-Einstein metric on $X_{c}$. The use of the same terminology for $\left(X_{0}, g_{0}\right)$ is however justified by the following equivalent definition. Before its formulation note that for the case of $\left(X_{c}, g_{c}\right)$ a complex submanifold $M \subset X_{c}$ is invariantly geodesic if and only if $\varphi(M) \subset X_{c}$ is totally geodesic in $\left(X_{c}, g_{c}\right)$ for any $\varphi \in \operatorname{Aut}\left(X_{c}\right)$. We have

Definition 3'. Let $\left(X_{0}, g_{0}\right)$ be a Hermitian symmetric space of the noncompact type, $\left(X_{c}, g_{c}\right)$ be its dual Hermitian symmetric space of the compact type, and $X_{0} \subset X_{c}$ be the Borel embedding. Then, a complex submanifold $S \subset X_{0}$ is an invariantly geodesic subspace if and only if $\varphi(S) \cap X_{0}$ is totally geodesic in $\left(X_{0}, g_{0}\right)$ whenever $\varphi \in \operatorname{Aut}\left(X_{c}\right)$ and $\varphi(S) \cap X_{0} \neq \emptyset$.

The equivalence between Definition 3' and Definition 3 follows readily from the proof of Tsai [Ts, Lemma (4.3)], which gives a characterization of invariantly geodesic subspaces in Lie-theoretic terms. Since we do not really need this equivalence here, 
except for a justification of the terminology in Definition 3, we omit the proof of the equivalence of the two definitions and refer the reader to [Ts].

(1.2) We collect some basic facts about characteristic symmetric subspaces and invariantly geodesic subspaces of Hermitian symmetric spaces of the compact or the noncompact type relevant to our discussion on proper holomorphic mappings between bounded symmetric domains. For the convenience of the reader we give an indication of the proof where appropriate.

Lemma 1. Let $X_{0}$ (resp. $X_{c}$ ) be an irreducible Hermitian symmetric space of the noncompact type (resp. compact type). Then, a characteristic symmetric subspace of $X_{0}\left(\right.$ resp. $\left.X_{c}\right)$ is an invariantly geodesic subspace in $X_{0}$ (resp. $X_{c}$ ).

Sketch of Proof. By Definition 3 it is sufficient to prove the lemma for the case of the compact type. Lemma 1 is stated for the case of $X_{c}$ in Tsai [Ts, §4, Examples 4.2(ii)]. Represent $X_{c}$ as a compatification of some $\mathbb{C}^{N}$ by means of Harish-Chandra coordinates. A totally geodesic complex submanifold $M$ of $\left(X_{c}, g_{c}\right)$ passing through $0 \in \mathbb{C}^{N}$ is the compactification of a complex vector subspace. Write $X_{c}=\operatorname{Aut}\left(X_{c}\right) / P$ where $\operatorname{Aut}\left(X_{c}\right)$ is the group of biholomorphic automorphisms of $X_{c}$ and $P \subset \operatorname{Aut}\left(X_{c}\right)$ is the parabolic subgroup. If $\varphi \in \operatorname{Aut}\left(X_{c}\right)$ is such that $\varphi(M) \cap \mathbb{C}^{N} \neq \emptyset$, then $\varphi=T_{a} \circ \gamma$ for some $\gamma \in P$ and for a translation $T_{a}, a \in \mathbb{C}^{N} ; T_{a}(z)=z+a$. In the case where $M \subset X_{c}$ is invariantly geodesic, $\gamma(M) \cap \mathbb{C}^{N}$ is a complex vector subspace for any $\gamma \in P$, so that $\varphi(M) \cap \mathbb{C}^{N}=T_{a}(\gamma(M)) \cap \mathbb{C}^{N}$ is affine-linear. Conversely, [Ts, Lemma (4.4)] shows that the affine-linearity of all $\varphi(M) \cap \mathbb{C}^{N} \neq \emptyset$ implies that $M \subset X_{c}$ is invariantly geodesic. The former property for a characteristic symmetric subspace $M \subset X_{c}$ resulted from Mok-Tsai [MT, Proposition 1.12].

Lemma 2. Let $X_{0}\left(\operatorname{resp} . X_{c}\right)$ be an irreducible Hermitian symmetric space of the noncompact type (resp. compact type). Let $\left\{S_{\alpha}\right\}_{\alpha \in A}$ be an arbitrary family of invariantly geodesic subspaces in $X_{0}$ (resp. $X_{c}$ ). Then, the intersection $S:=\bigcap_{\alpha \in A} S_{\alpha}$ is an invariantly geodesic subspace in $X_{0}$ (resp. $X_{c}$ ).

Proof. Obvious from Definitions 2 and 3 (or 3').

Lemma 3. Let $D(p, q)$ be a classical symmetric domain of type I represented by the set of p-by-q matrices $Z$ with complex coefficients such that $I-\bar{Z}^{t} Z$ is positive definite. For positive integers $s \leq p, t \leq q$, let $\tau: D(s, t) \hookrightarrow D(p, q)$ be the standard embedding. Then, any invariantly geodesic subspace $S$ is biholomorphically equivalent to $D(s, t)$ for some choice of $(s, t)$ and the implicit holomorphic embedding $\mu: S \hookrightarrow D(p, q)$ is equivalent to $\tau$ up to automorphisms of $D(s, t) \cong S$ and $D(p, q)$.

Proof. Lemma 3 follows from the classification of invariantly geodesic subspaces of the Grassmannian as given in Tsai [Ts, Proposition 4.6], which results from a classification of invariantly geodesic subspaces of the characteristic subvariety $\mathcal{S}_{0}(G(p, q))=\mathbb{P}^{p-1} \times$ $\mathbb{P}^{q-1}$.

Here by the standard embedding $\tau: D(s, t) \rightarrow D(p, q)$ we mean the mapping defined 
by $\tau(Z)=\left[\begin{array}{ll}Z & 0 \\ 0 & 0\end{array}\right]$. For an irreducible Hermitian symmetric space $X_{c}$ of the compact type, the characteristic subvariety $\mathcal{S}_{0}\left(X_{c}\right) \subset \mathbb{P} T_{0}\left(X_{c}\right)$ is the set of projectivizations of characteristic vectors. The latter are equivalently non-zero vectors tangent to minimal rational curves. In other words, $\mathcal{S}_{0}\left(X_{c}\right)$ is the variety of minimal rational tangents at $0 \in X_{c}$ (cf. Hwang-Mok [HM, §1]).

Lemma 4. Let $\Omega$ and $\Omega^{\prime}$ be irreducible bounded symmetric domains equipped with canonical Kähler-Einstein metrics normalized such that minimal disks are of constant Gaussian curvature -1. Suppose $\Omega$ is of rank $\geq 2$. Let $U \subset \Omega$ be a nonempty open subset and $f: U \rightarrow \Omega^{\prime}$ be a holomorphic embedding such that up to a normalizing constant $f$ is an isometry on characteristic vectors. Then, $f: U \rightarrow \Omega^{\prime}$ is a totally geodesic isometric embedding.

Proof. From the hypothesis it follows by the polarization argument of Mok [Mk2, (3.2), proof of $(\mathrm{i}) \Rightarrow($ ii)] that $f$ is an isometry up to a global constant. Let $(\alpha, \zeta)$ be a zero of holomorphic bisectional curvature at $x \in U$, i.e., $R_{\alpha \bar{\alpha} \zeta \bar{\zeta}}=0$ for the curvature $R$ of $\Omega$ with respect to the canonical Kähler-Einstein metric. Since $f$ is a holomorphic isometry up to a normalizing constant we also have $R_{\alpha \bar{\alpha} \zeta \bar{\zeta}}^{\prime}=0$, where $R^{\prime}$ is the curvature tensor of $\Omega^{\prime}$ with respect to the canonical Kähler-Einstein metric, and $\alpha$ is identified with $d f(\alpha)$, etc. By the Gauss Lemma it follows that $\sigma(\alpha, \zeta)=0$ for the second fundamental form $\sigma$ of the holomorphic embedding $f: U \rightarrow \Omega^{\prime}$. By the polarization argument as in Mok [Mk1, proof of Proposition (3.4)] it follows that $f$ is totally geodesic. Since minimal disks are mapped biholomorphically onto minimal disks by such a map $f$, by the choices of normalization on canonical Kähler-Einstein metrics it follows that $f$ is a bona fide isometry without any normalizing constant.

Next, we collect here preceding rigidity and nonexistence results on proper holomorphic mappings between bounded symmetric domains.

Theorem A (Tsai [Ts, Main Theorem]). Let $f: \Omega \rightarrow \Omega^{\prime}$ be a proper holomorphic map between two bounded symmetric domains such that $\Omega$ is irreducible and of rank $\geq 2$, and such that $\operatorname{rank}\left(\Omega^{\prime}\right) \leq \operatorname{rank}(\Omega)$. Then, $\operatorname{rank}\left(\Omega^{\prime}\right)=\operatorname{rank}(\Omega)$, and $f: \Omega \rightarrow \Omega^{\prime}$ is a totally geodesic embedding.

Theorem B (Tu [Tu1, Theorem 1.1]). Let $\Omega$ be an irreducible bounded symmetric domain of rank $\geq 2$. Suppose $\Omega^{\prime}$ is a bounded symmetric domain, $\operatorname{dim}\left(\Omega^{\prime}\right)=\operatorname{dim}(\Omega)$. Then, any proper holomorphic map $f: \Omega \rightarrow \Omega^{\prime}$ is a biholomorphism.

The result that any proper holomorphic self-map on an irreducible bounded symmetric domain $\Omega$ of rank $\geq 2$ is necessarily a biholomorphic automorphism was due to Henkin-Novikov [HN]. We note that Tu [Tu3] contains a survey on related results about proper holomorphic maps between bounded symmetric domains.

The first of the series of classical domains of E. Cartan is given by

$$
D(p, q)=\left\{Z \in M(p, q, \mathbb{C}): I-\bar{Z}^{t} Z>0\right\}, \quad p, q \geq 1
$$


$D(p, q)$ is of rank $r=\min (p, q)$. If $p \geq q \geq 2$, then $r=q$, and the characteristic symmetric subspaces are up to automorphisms of $D(p, q)$ given by $D(p-k, q-k)$, $1 \leq k \leq q-1$. In what follows we are concerned exclusively with the study of proper holomorphic mappings between Cartan domains of type I, although it is possible to generalize the nonexistence results to certain other pairs of irreducible bounded symmetric domains. Concerning $D(p, q)$ we have the following result of $\mathrm{Tu}[\mathrm{Tu} 2]$ which gives the only known nontrivial rigidity and nonexistence results for proper holomorphic maps between bounded symmetric domains in the non-equirank case.

Theorem C (Tu [Tu2, Theorem 1.1 and Corollary 1.2]). Let $p \geq 3$. Then, any proper holomorphic map $f: D(p, p-1) \rightarrow D(p, p)$ is a totally geodesic embedding. As a consequence, there does not exist any proper holomorphic mapping from $D(p+1, p-1)$ into $D(p, p)$.

We note that the rank defect is always equal to 1 in the result. The main purpose of the current article is to give nonexistence results for arbitrarily large co-ranks, and to motivate the formulation of questions on the subject at the end of the article. We will give a proof of the nonexistence result in Theorem $\mathrm{C}$ without using the rigidity result for the case of $p \geq 4$ by resorting to the geometry of invariantly geodesic subspaces. This proof will then be generalized in a way that avoids establishing an analogous rigidity result for arbitrarily prescribed rank defects, a problem hitherto unresolved which has remained technically difficult along the line of approach of [Tu2].

\section{$\S 2$ Nonexistence results for proper holomorphic mappings between bounded symmetric domains}

(2.1) The Main Theorem of this article will give a nonexistence result with prescribed rank defects for proper holomorphic mappings between Cartan domains of type I. To start with, we give a proof of the nonexistence result of $\mathrm{Tu}$ [Tu2] (Theorem $\mathrm{C}$ in (1.1) here) for the case of $p \geq 4$ which leads to an effective nonexistence result for the case where the rank defect is equal to 2 .

\section{Proposition 1.}

(a) For $p \geq 3$ there does not exist any proper holomorphic mapping from $D(p+1, p-1)$ into $D(p, p)(\mathrm{Tu}[\mathrm{Tu} 2])$.

(b) For $p \geq 7$ there does not exist any proper holomorphic mapping from $D(p+2, p-2)$ into $D(p, p)$.

Proof. We first give a proof of (a) for $p \geq 4$ by a method which does not rely on the rigidity result of $\mathrm{Tu}$ for proper holomorphic mappings from $D(p, p-1)$ to $D(p, p)$. A modification of the proof will yield (b).

Proof of (a) for $p \geq 4$. We are going to argue by contradiction. Let $f=D(p+1, p-1) \rightarrow$ $D(p, p)$ be a hypothetical proper holomorphic map. Let $\Pi \subset D(p+1, p-1)$ be any characteristic symmetric subspace of co-rank 1 (i.e., of rank $p-2), \Pi \cong D(p, p-2)$. $\mathrm{By}$ Proposition $\left.f\right|_{\Pi}: \Pi \rightarrow D(p, p)$ is a proper holomorphic map whose image lies in some proper characteristic symmetric subspace $\Pi^{\prime}$ of $D(p, p)$. Without loss of generality 
we may assume that $\Pi^{\prime} \subset D(p, p)$ is of co-rank 1 (i.e., of rank $p-1$ ). Thus, up to automorphisms of $D(p, p-2)$ resp. $D(p, p),\left.f\right|_{\Pi}: \Pi \rightarrow \Pi^{\prime}$ is equivalent to a proper holomorphic map $g: D(p, p-2) \rightarrow D(p-1, p-1)$. We identify $\Pi$ with $D(p, p-2) \hookrightarrow$ $D(p+1, p-1)$. In what follows the superscript $r$ in the notation of a vector space $U^{(r)}$ indicates its dimension.

Identify now $T_{0}(D(m, n)) \cong M(m, n ; \mathbb{C}) \cong \mathbb{C}^{m} \otimes \mathbb{C}^{n}$ in the usual way. In our case we identify $T_{0}(D(p+1, p-1))$ with $E^{(p+1)} \otimes F^{(p-1)}$, where $E^{(p+1)} \cong \mathbb{C}^{p+1}$, etc. Then $T_{0}(D(p, p-2))=V^{(p)} \otimes W^{(p-2)}$; where $V^{(p)} \subset E^{(p+1)}, W^{(p-2)} \subset F^{(p-1)}$. Let $S^{(p-1)} \subset V^{(p)}$ be a general $(p-1)$-dimensional vector subspace. In other words, the point $\left[S^{(p-1)}\right]$ in the Grassmannian $\operatorname{Gr}\left(p-1, V^{(p)}\right)$ of $(p-1)$-dimensional vector subspaces is a general point in the sense of Algebraic Geometry, i.e. lying outside some proper complex-analytic subvariety of $\operatorname{Gr}\left(p-1, V^{(p)}\right)$. This notion of a general point in a moduli space will be used in the sequel without further explanation. Consider the totally geodesic Hermitian symmetric subspace $\Xi \subset \Pi, \Xi \cong D(p-1, p-2)$, such that $T_{0}(\Xi)$ is canonically identified with $S^{(p-1)} \otimes W^{(p-2)}$. (Note that $\Xi \subset \Pi \cong D(p, p-2)$ is not a characteristic symmetric subspace). For any $p$-dimensional vector subspace $\widetilde{V}^{(p)} \supset S^{(p-1)}$, and for the characteristic symmetric subspace $\widetilde{\Pi} \subset D(p+1, p-1)$ such that $T_{0}(\widetilde{\Pi})=\widetilde{V}^{(p)} \otimes W^{(p-2)}$, we have the proper holomorphic map $\left.f\right|_{\widetilde{\Pi}}: \widetilde{\Pi} \rightarrow D(p, p)$ such that $f(\widetilde{\Pi})$ is contained in a proper characteristic symmetric subspace $\widetilde{\Pi}^{\prime} \subset D(p, p)$. We may write $\widetilde{\Pi}=\widetilde{\Pi}\left(\widetilde{V}^{(p)}\right), \widetilde{\Pi}^{\prime}=\widetilde{\Pi}^{\prime}\left(\widetilde{V}^{(p)}\right)$; and we have

$$
\begin{gathered}
f(\Xi)=f\left(\left\{\bigcap \widetilde{\Pi}\left(\widetilde{V}^{(p)}\right): \widetilde{V}^{(p)} \supset S^{(p-1)}\right\}\right) \\
=\bigcap\left\{f\left(\widetilde{\Pi}\left(\widetilde{V}^{(p)}\right)\right): \widetilde{V}^{(p)} \supset S^{(p-1)}\right\} \subset \bigcap\left\{\widetilde{\Pi}^{\prime}\left(\widetilde{V}^{(p)}\right): \widetilde{V}^{(p)} \supset S^{(p-1)}\right\} .
\end{gathered}
$$

Each $\widetilde{\Pi}^{\prime}\left(\widetilde{V}^{(p)}\right)$ is a characteristic symmetric subspace of $D(p, p)$, in particular, an invariantly geodesic subspace of $D(p, p)$, by Lemma 1. By Lemma 2, their intersection $\Phi_{0} \subset D(p, p)$ is again an invariantly geodesic subspace. By Lemma 3, we have $f(\Xi) \subset \Phi_{0} \subset D(p, p)$, where $\Phi_{0}$ is up to automorphisms of $D(p, p)$ the standard $D(s, t) \hookrightarrow D(p, p)$. Thus, we have one of the two alternatives. Either we may take

( $\alpha) \widetilde{\Pi}^{\prime}\left(\widetilde{V}^{(p)}\right)=\widetilde{\Pi}^{\prime}\left(V^{(p)}\right)=D(p-1, p-1) \subset D(p, p)$ for each $\widetilde{V}^{(p)} \supset S^{(p-1)}$; or

$(\beta) \Phi_{0} \subset \Phi \varsubsetneqq D(p-1, p-1)$, and $\Phi$ is up to left and right multiplication by unitary matrices equivalent to $D(p-1, p-2)$ or $D(p-2, p-1)$.

For Alternative $(\alpha)$ consider the totally geodesic Hermitian symmetric subspace $\Psi \subset$ $D(p+1, p-1)$ such that $T_{0}(\Psi)=E^{(p+1)} \otimes W^{(p-2)}$. Note that any two distinct $p$ dimensional vector subspaces in $E^{(p+1)}$ interest in a $(p-1)$-dimensional vector subspace of $E^{(p+1)}$. Since by assumption $S^{(p-1)} \subset V^{(p)}$ is a general $(p-1)$-dimensional linear subspace, Alternative $(\alpha)$ implies that $f(\widehat{\Pi}) \subset \Pi^{\prime}$ for a general characteristic symmetric subspace $\widehat{\Pi} \subset D(p+1, p-1)$ of co-rank 1 such that $0 \in \Pi$ and $T_{0}(\widehat{\Pi})=\widehat{V}^{(p)} \otimes W^{(p-2)}$, $\widehat{V}^{(p)} \subset E^{(p+1)}$. By continuity it follows that $f(\widehat{\Pi}) \subset \Pi^{\prime}$ for every such characteristic symmetric subspace $\widehat{\Pi}$. Since the union of such $\widehat{\Pi}$ exhausts $\Psi$, we deduce $f(\Psi) \subset$ 
$\widetilde{\Pi}^{\prime}\left(V^{(p)}\right)=\Pi^{\prime}=D(p-1, p-1)$. But

$$
\begin{gathered}
\operatorname{dim}(\Psi)=(p+1)(p-2)=p^{2}-p-2 ; \operatorname{dim}\left(\Pi^{\prime}\right)=(p-1)^{2}=p^{2}-2 p+1 ; \\
\operatorname{dim}(\Psi)>\operatorname{dim}\left(\Pi^{\prime}\right) \Leftrightarrow p^{2}-p-2>p^{2}-2 p+1 \Leftrightarrow p>3 .
\end{gathered}
$$

Thus for $p \geq 4, \operatorname{dim}(\Psi)>\operatorname{dim}\left(\Pi^{\prime}\right)$, and there does not exist any proper holomorphic mapping from $\Psi$ into $\Pi$. To prove (a) it remains to get a contradiction to the hypothetical existence of a proper holomorphic map $f: D(p+1, p-1) \rightarrow D(p, p)$ in Alternative $(\beta)$. In this case we have a proper holomorphic map $\left.f\right|_{\Xi}: \Xi \rightarrow \Phi \subset D(p, p)$ which is equivalent to a proper holomorphic map $h: D(p-1, p-2) \rightarrow D(p-1, p-2)$. By HenkinNovikov $[\mathrm{HN}]$ any such holomorphic map must be a biholomorphism. For $p \geq 4$, if Alternative $(\alpha)$ occurs for some choice of $\Pi \subset D(p+1, p-1), \Pi \cong D(p, p-2)$, we already have a contradiction. We may therefore assume that Alternative $(\beta)$ holds true for any choice of characteristic symmetric subspace $\Pi \subset D(p+1, p-1)$ of co-rank 1 passing through 0 . It follows that for any $(p-2)$-dimensional vector subspace $W^{(p-2)} \subset F^{(p-1)}$, and any general $(p-1)$-dimensional vector subspace $S^{(p-1)} \subset E^{(p+1)}$, taking $\Xi \cong D(p-1, p-2)$ to be such that $T_{0}(\Xi)=S^{(p-1)} \otimes W^{(p-2)},\left.f\right|_{\Xi}: \Xi \rightarrow \Phi \subset D(p, p)$ must necessarily be a totally geodesic embedding. By continuity it remains the case that $\left.f\right|_{\Xi}: \Xi \rightarrow D(p, p)$ is a totally geodesic embedding without assuming that $S^{(p-1)} \subset E^{(p+1)}$ is a general $(p-1)$-dimensional vector subspace. Let now $\eta \in T_{0}(D(p+1, p-1))$ be an arbitrary characteristic vector. Choosing $\Xi \cong D(p-1, p-2)$ as in the above such that $\eta \in T_{0}(\Xi)$ we have a fortiori $\|d f(\eta)\|=\|\eta\|$ with respect to norms of canonical Kähler-Einstein metrics on the bounded symmetric domains $D(k, \ell)$, where we fix the normalizing constants by agreeing that minimal disks are of constant Gaussian curvature -1 . Since any point $x \in D(p+1, p-1)$ can be transformed to 0 by an automorphism, it follows that $f: D(p+1, p-1) \rightarrow D(p, p)$ is a holomorphic isometry on characteristic vectors. By Lemma $4, f$ is a totally geodesic holomorphic embedding, which is absurd.

Proof of (b). We now adapt the proof of (a) for $p \geq 4$ to give a proof of (b). Considering now a hypothetical proper holomorphic map $f: D(p+2, p-2) \rightarrow D(p, p)$ (in place of $f: D(p+1, p-1) \rightarrow D(p, p))$ and using exactly the same set-up as in (a) together with obvious interpretations of the notations we arrive at the following alternatives. Either we may take

$(\alpha) \widetilde{\Pi}^{\prime}\left(\widetilde{V}^{(p+1)}\right)=\widetilde{\Pi}^{\prime}\left(V^{(p+1)}\right)=D(p-1, p-1) \subset D(p, p)$ for each $\widetilde{V}^{(p+1)} \supset S^{(p)}$; or

( $\beta) \Phi_{0} \subset \Phi \varsubsetneqq D(p-1, p-1)$, and $\Phi$ is up to left and right multiplication by unitary matrices equivalent to $D(p-1, p-2)$ or $D(p-2, p-1)$.

For Alternative $(\alpha)$ consider the totally geodesic Hermitian symmetric subspace $\Psi \subset$ $D(p+2, p-2)$ such that $T_{0}(\Psi)=E^{(p+2)} \otimes W^{(p-3)}$. Then $f(\Psi) \subset \widetilde{\Pi}^{\prime}\left(V^{(p+1)}\right)=\Pi^{\prime}=$ $D(p-1, p-1)$. But

$$
\begin{gathered}
\operatorname{dim}(\Psi)=(p+2)(p-3)=p^{2}-p-6 ; \operatorname{dim}\left(\Pi^{\prime}\right)=(p-1)^{2}=p^{2}-2 p+1 ; \\
\operatorname{dim}(\Psi) \geq \operatorname{dim}\left(\Pi^{\prime}\right) \Leftrightarrow p^{2}-p-6 \geq p^{2}-2 p+1 \Leftrightarrow p \geq 7 .
\end{gathered}
$$

For $p \geq 8, \operatorname{dim}(\Psi)>\operatorname{dim}\left(\Pi^{\prime}\right)$ and there cannot be any proper holomorphic map $\left.f\right|_{\Psi}$ : $\Psi \rightarrow \Pi^{\prime}$. For $p=7,\left.f\right|_{\Psi}$ is equivalent to a proper holomorphic map $h: D(9,4) \rightarrow D(6,6)$ 
in which the domain and target spaces are equidimensional and biholomorphically distinct. By Tu [Tu1], such proper holomorphic mapping does not exist.

Thus for (b) we are reduced to Alternative $(\beta)$ where we have obtained, in analogy to (a), proper holomorphic mappings $\left.f\right|_{\Xi}: \Xi \rightarrow \Phi_{0} \varsubsetneqq D(p-1, p-1) \subset D(p, p)$ where $T_{0}(\Xi)=S^{(p)} \otimes W^{(p-3)}$, thus $\Xi \cong D(p, p-3)$, and $\Phi_{0} \subset \Phi \cong D(p-1, p-2)$. We have thus equivalently a proper holomorphic mapping $h: D(p, p-3) \rightarrow D(p-1, p-2)$. Here

$$
\begin{gathered}
\operatorname{dim}(D(p, p-3))=p(p-3)=p^{2}-3 p<p^{2}-3 p+2 \\
=(p-1)(p-2)=\operatorname{dim}(D(p-1, p-2)) ; \\
\operatorname{rank}(D(p, p-3))=p-3<p-2=\operatorname{rank}(D(p-1, p-2))
\end{gathered}
$$

and it is not clear that such a proper holomorphic map cannot exist. To reach a contradiction we have to repeat the reduction argument in (a) again. Starting with a proper holomorphic map $h: D(p, p-3) \rightarrow D(p-1, p-2)$ and repeating the arguments of (a) in order to rule out the analogue of $(\alpha)$ we have to show that there does not exist any proper holomorphic mapping from $D(p, p-4)$ into $D(p-2, p-3)$. For this it suffices to verify that $\operatorname{dim}(D(p, p-4))>\operatorname{dim}(D(p-2, p-3))$ i.e., $p^{2}-4 p>p^{2}-5 p+6$, which holds true if and only if $p>6$, while we assumed $p \geq 7$. We are then reduced to ruling out the analogue of $(\beta)$ where we obtain a proper holomorphic mapping $h^{\prime}$ from $D(p-2, p-4)$ into a proper invariantly geodesic subspace of $D(p-2, p-3)$. Without loss of generality we may assume the target space to be either $D(p-2, p-4)$ or $D(p-3, p-3)$. In the case of $h^{\prime}: D(p-2, p-4) \rightarrow D(p-2, p-4)$ the arguments in (a) using Henkin-Novikov $[\mathrm{HN}]$ and Lemma 4 apply to derive a contradiction to the existence of $h: D(p, p-3) \rightarrow D(p-1, p-2)$, where for the argument we take all possible $h^{\prime}$ (and not just a single map) obtained from $h$ by restriction in analogy to the argument in (a). In the case of $h: D(p-2, p-4) \rightarrow D(p-3, p-3)$ we are back to (a) of Proposition 1, where $h: D(q+1, q-1) \rightarrow D(q, q)$ with $q=p-3 \geq 4$. By (a) such a proper holomorphic map $h$ cannot exist. The proof of Proposition 1 is completed.

REMARKS. In the proof we have used implicitly the fact that maps induced from $f$ between moduli spaces of invariantly geodesic subspaces (including the special case of characteristic symmetric subspaces) induced from the proper holomorphic map $f: \Omega \rightarrow$ $\Omega^{\prime}$ under consideration are meromorphic (cf. Mok-Tsai [MT, proof of Proposition 2.3]) for a proof in a similar situation). .

(2.2) In relation to the study of proper holomorphic mappings $f: \Omega \rightarrow \Omega^{\prime}$ between irreducible bounded symmetric domains, especially in the case of Type-I domains of E. Cartan, in principle the difficulty increases with the co-rank of the pair $\left(\Omega, \Omega^{\prime}\right)$, i.e., $\operatorname{rank}\left(\Omega^{\prime}\right)-\operatorname{rank}(\Omega)$, noting that by Tsai $[\mathrm{Ts}]$ no such maps $f: \Omega \rightarrow \Omega^{\prime}$ can exist when $\operatorname{rank}\left(\Omega^{\prime}\right)<\operatorname{rank}(\Omega)$, and that $f$ must be a totally geodesic embedding in the equal rank case. Tu $[\mathrm{Tu} 1,2]$ obtained both rigidity and nonexistence results in some cases of co-rank 1. Proposition 1 extends nonexistence results in some cases of co-rank 2. The proof of Proposition 1 can be adapted to yield nonexistence results for examples of pairs $\left(\Omega, \Omega^{\prime}\right)$ of arbitrarily large co-ranks. The structure of the proof in the case of co-rank 2 
also suggests that we should introduce a reduction argument in a more general context. We state our result in a qualitative way, as follows.

Main Theorem. Let $k, \ell$ be integers, $k \geq 0, \ell>0$. Then, there exist a positive integer $N(k, \ell)$ such that for any integer $p \geq N(k, \ell)$, there does not exist any proper holomorphic map $f: D(p+k+\ell, p-\ell) \rightarrow D(p+k, p)$.

Proof. Assume that $f$ exists. Imitating the reduction argument in the proof of Proposition 1 in the case (a); where $k=0, \ell=1$, and $p \geq 4$; we derive two alternatives. Either

$(\alpha)$ there exists a proper holomorphic map $g: D(p+k+\ell, p-\ell-1) \rightarrow D(p+k-1, p-1)$; or

$(\beta)$ there exists a proper holomorphic map $h: D(p+k+\ell-2, p-\ell-1) \rightarrow D(p+k-2, p-1)$ or $D(p+k-1, p-2)$

Alternative $(\alpha)$ does not occur provided that $\operatorname{dim}(D(p+k+\ell, p-\ell-1))>\operatorname{dim}(D(p+$ $k-1, p-1))$. In other words, we require

$$
\begin{gathered}
(p+k+\ell)(p-\ell-1)>(p+k-1)(p-1), \quad \text { i.e. }, \\
p^{2}+(k-1) p-(k+\ell)(\ell+1)>p^{2}+(k-2) p+1-k, \quad \text { i.e. }, \\
p>(k+\ell)(\ell+1)+1-k=\ell^{2}+(k+1) \ell+1 .
\end{gathered}
$$

We are left with Alternative $(\beta)$. To prove the Main Theorem we have to do a reduction argument on the pair of indices $(k, \ell)$ with $k \geq 0, \ell>0$. If $k>0$, in Alternative $(\beta)$ we have either

$$
\begin{gathered}
h: D(q+(k-1)+\ell, q-\ell) \rightarrow D(q+(k-1), q), q=p-1 ; \quad \text { or } \\
h: D(q+(k+1)+(\ell-1), q-(\ell-1)) \rightarrow D(q+(k+1), q), q=p-2 .
\end{gathered}
$$

For $k \geq 0, \ell>0, p \geq \ell+2$ we denote by $\Phi(k, \ell ; p)$ the statement that there does not exist any proper holomorphic map $f: D(p+k+\ell, p-\ell) \rightarrow D(p+k, p)$. At the same time $\Phi(k, 0 ; p), p \geq 2$, is the statement that any proper holomorphic map $f: D(p+k, p) \rightarrow D(p+k, p)$ is a biholomorphism. Thus $\Phi(k, 0 ; p), p \geq 2$, always holds true by Henkin-Novikov [HN]. Given $(k, \ell ; p), k, \ell \geq 0, p>\ell+2$, we need to show that there exists $N(k, \ell)$ such that $\Phi(k, \ell ; p)$ holds true whenever $p \geq N(k, \ell)$. For $\ell=0$ we just take $N(k, 0)=2$. Our argument so far says for $k, \ell>0, p \geq 4$

(†) If $p>\ell^{2}+(k+1) \ell+1$, then $\Phi(k, \ell ; p)$ holds true whenever both $\Phi(k-1, \ell ; p-1)$ and $\Phi(k+1, \ell-1 ; p-2)$ hold true.

In the case $k=0, \ell>0, p \geq 4$ there is a modification. Alternative $(\beta)$ for $k=0$ corresponds to having a proper holomorphic map

$$
\begin{gathered}
h: D(p+\ell-2, p-\ell-1) \rightarrow D(p-1, p-2) ; \quad \text { i.e }, \\
h: D(q+(k+1)+(\ell-1), q-(\ell-1)) \rightarrow D(q+1, q), q=p-2 .
\end{gathered}
$$

We have thus

$(\dagger)_{0}$ If $p>\ell^{2}+\ell+1$, then $\Phi(0, \ell ; p)$ holds true whenever $\Phi(1, \ell-1 ; p-2)$ holds true. 
Given $k \geq 0, \ell>0$, the validity of $\Phi(k, \ell ; p)$ will follow for $p$ sufficiently large, if in the reduction process as given either by $(\dagger)$ or $(\dagger)_{0}$ we eventually reach $(k, 0)$. In $(\dagger)$ we move from $(k, \ell)$ to either $(k-1, \ell)$ or $(k+1, \ell-1)$. We call these moves of the first resp. second kind. We want to show that $(k, \ell)$ is always moved to $\left(k^{\prime}, 0\right)$, in a number of moves whose cardinality is bounded in terms of $(k, \ell)$. Observe that in each move $\ell$ is either fixed or reduced by 1 . Note, by $(\dagger)_{0}$, that $(0, \ell)$ is always moved to $(1, \ell-1)$. Starting with $(k, \ell)$ in at most $k+1$ steps $\ell$ is forced to be reduced by 1 . Since the sum of the two indices is either fixed or reduced by 1 in each move, it follows readily that for some integer $\theta(k, \ell)$, depending on $(k, \ell),(k, \ell)$ must be moved to some $\left(k^{\prime}, 0\right)$ in at most $\theta(k, \ell)$ moves. Actually we may take $\theta(k, \ell)=k+2 \ell$. In fact, there are $\ell$ moves of the second kind, which reduce the second index. In each of these $\ell$ moves the first index is increased by 1 , and there are precisely $k+\ell-k^{\prime}$ moves of the first kind so that the first index finally becomes $k^{\prime}$, as a consequence of which there are altogether $\ell+\left(k+\ell-k^{\prime}\right) \leq k+2 \ell$ moves involved.

Given $(k, \ell)$ we choose $p$ large enough so that $\Phi(k, \ell ; p)$ can be reduced in at most $k+2 \ell$ steps to a statement $\Phi\left(k^{\prime}, 0 ; r\right)$ with $r \geq 2$. For this purpose it suffices to choose $p$ sufficiently large so that

(i) in each step the dimension requirement ruling out Alternative $(\alpha)$ is satisfied, allowing us to reduce to Alternative $(\beta)$;

(ii) $p \geq 2(k+2 \ell)+2=2 k+4 \ell+2$ so that after $k+2 \ell$ steps $\Phi(k, \ell ; p)$ is reduced to a statement $\Phi\left(k^{\prime}, 0 ; r\right)$ with $r \geq 2$. Here $p \geq 2 k+4 \ell+2$ suffices since in Alternative $(\beta)$, the index $p$ is in each step either reduced by 1 or 2 .

It is clear that there exists some integer $N(k, \ell)$ such that (i) and (ii) are satisfied whenever $p \geq N(k, \ell)$. The proof of the Main Theorem is completed.

Remarks. Clearly the Main Theorem can be made effective as $N(k, \ell)$ can be estimated. For instance, for $k=0$ we can take $N(0, \ell)$ to be $O\left(\ell^{2}\right)$. We will refrain from getting an optimal estimate on $N(k, \ell)$ from the method of the article, as the primary purpose of the Main Theorem is to produce examples of nonexistence results on proper holomorphic maps in which the rank defects are arbitrary prescribed positive integers.

(2.3) The nonexistence result Theorem 1 of the current article gives examples of pairs of irreducible bounded symmetric domains of an arbitrarily prescribed co-rank for which there does not exist any proper holomorphic mappings. It serves as a motivation for formulating the following questions regarding proper holomorphic mappings between bounded symmetric domains.

Question 1. Let $\Omega$ and $\Omega^{\prime}$ be bounded symmetric domains such that $\Omega$ is irreducible and of rank $\geq 2$. Suppose there exists a proper holomorphic map $f: \Omega \rightarrow \Omega^{\prime}$. Does there always exist a totally geodesic holomorphic embedding $h: \Omega \rightarrow \Omega^{\prime}$ ?

QUESTION 2. In the notations of Question 2 suppose there exists a proper holomorphic mapping $f: \Omega \rightarrow \Omega^{\prime}$ which is not totally geodesic. Is $\Omega$ necessarily biholomorphically equivalent to a characteristic symmetric subspace of $\Omega^{\prime}$ ?

Both Question 1 and 2 for Type-I domains $\Omega=D(p, q)$ of E. Cartan, $\Omega^{\prime}=D\left(p^{\prime}, q^{\prime}\right)$ 
are already very interesting. The nonexistence result in Theorem 1 on series of pairs $\Omega=D(p, q), \Omega^{\prime}=D\left(p^{\prime}, q^{\prime}\right)$ is in agreement with a positive answer to Question 1 , but at this point there is not enough evidence why one should expect the answers to Question 1 and 2 to be positive even for Type-I domains. At the same time if there are counterexamples to either Question 1 or Question 2, especially the latter, it appears that Type-I domains of E. Cartan are the most plausible places to look for them.

\section{References}

[HM] Hwang, J.-M. and Mok, N. Holomorphic maps from rational homogeneous spaces of Picard number 1 onto projective manifolds, Invent. Math., 136 (1999), 209331.

[HN] Henkin, G.M. and Novikov, R. Proper mappings of classical domains, in Linear and Complex Analysis Problem Book, Lecture Notes in Math. Vol. 1043, Springer, Berlin, 1984, pp.625-627.

[Mk1] Mok, N. Uniqueness theorem of Hermitian metrics of seminegative curvature on quotients of bounded symmetric domains, Ann. Math. 125 (1987), 105-152.

[Mk2] Mok, N. Metric Rigidity Theorems on Hermitian Locally symmetric spaces, Series in Pure Math. Vol. 6, World Scientific, Singapore, 1989.

[MT] Mok, N.; Tsai, I.-H. Rigidity of convex realizations of irreducible bounded symmetric domains of rank2, J. Reine Angew. Math. 431(1992), 91-122.

[Si] Siu, Y.-T. The complex analyticity of harmonic maps and the strong rigidity of compact Kähler manifolds, Amer. J. Math. 100(1978), 197-203.

[Ts] Tsai, I.-H. Rigidity of proper holomorphic maps between symmetric domains, J. Diff. Geom. 37(1993), 123-160.

[Tu1] Tu, Z.-H. Rigidity of proper holomorphic mappings between equidimensional bounded symmetric domains, Proc. Amer. Math. Soc. 130(2002), 1035-1042.

[Tu2] Tu, Z.-H. Rigidity of proper holomorphic mappings between nonequidimensional bounded symmetric domains, Math. Z. 240(2002), 13-35.

[Tu3] Tu, Z.-H. Rigidity of proper holomorphic mappings between bounded symmetric domains, Geometric function theory in several complex variables, pp.310-316, World Sci. Publishing, River Edge, NJ, 2004.

[Wo] Wolf, J. A. Fines structure of Hermitian symmetric spaces, in Geometry of Symmetric Spaces, ed. Boothby-Weiss, Marcel-Dekker, New York, 1972, pp.271357.

Department of Mathematics

The University of Hong Kong

Pokfulam Road

Hong Kong

Email: nmok@hku.hk 